\title{
On Inter-Expressibility of Logical Connectives in Gödel Fuzzy Logic
}

\author{
Vítězslav Švejdar*† Kamila Bendová
}

June 2, 1999

The original publication is available at www.springerlink.com.

\begin{abstract}
In Gödel fuzzy propositional logic, neither conjunction nor implication is expressible (definable) in terms of the remaining three logical connectives.
\end{abstract}

In classical propositional logic, propositions are associated with two truth values 1 (truth) and 0 (falsity). In fuzzy logics further (intermediate) truth values lying between 0 and 1 are possible. Usually the truth values are linearly ordered and it is common to assume that they are elements of the real interval $[0,1]$.

In Gödel fuzzy propositional logic G (see Gödel [5] and/or Hájek [6]), we deal with propositional formulas built up from (say denumerable infinite set of) propositional atoms using the logical connectives \& (conjunction), $\vee$ (disjunction), $\rightarrow$ (implication) and $\neg$ (negation). The meaning of the four logical connectives is determined by their truth functions defined as follows. The truth function of conjunction and disjunction are the functions min (minimum) and $\max$ (maximum). The truth function of implication is the residuum function $\Rightarrow$, where $x \Rightarrow y=y$ if $y<x$ and $x \Rightarrow y=1$ otherwise. The truth function of negation is the function - defined by $-x=x \Rightarrow 0$ (so, in this paper, the symbol - does not denote subtraction). The real interval $[0,1]$ equipped with the functions $\min , \max , \Rightarrow$ and - is called the standard $\mathrm{G}$-algebra and denoted $[0,1]_{\mathrm{G}}$.

A truth evaluation is a function from propositional atoms to $[0,1]$. In full analogy to the classical case, any truth evaluation has a uniquely determined extension defined on all propositional formulas and respecting the truth functions of the standard G-algebra defined above. A propositional formula $A$ is a G-tautology if $v(A)=1$ for each truth evaluation $v$. An example

${ }^{*}$ Charles University, Prague, and Institute of Computer Science of the Academy of Sciences of the Czech Republic, Prague, vitezslavdotsvejdaratcunidotcz, http://www1.cuni.cz/ svejdar/.

${ }^{\dagger}$ This paper was supported by grant 401/98/0383 of the Grant Agency of the Czech Republic.

$\ddagger$ Charles University, Prague. 
of a G-tautology is any formula of the form $\neg A \vee \neg \neg A$ : if $v(A) \neq 0$ then $v(\neg A)=0$ and $v(\neg \neg A)=1$, if $v(A)=0$ then $v(\neg A)=1$; in both cases $\max (v(\neg A), v(\neg \neg A))=1$. An example of a formula that is not a G-tautology is $\neg \neg p \rightarrow p$ : for $v$ satisfying $v(p)=\frac{1}{2}$ we have $v(\neg \neg p \rightarrow p)=1 \Rightarrow \frac{1}{2}=\frac{1}{2}$. Clearly any $\mathrm{G}$-tautology is a classical tautology.

A common way to construct a Hilbert (or Frege) style calculus for the logic G is to take a Hilbert calculus for intuitionistic propositional logic (e.g. the version from [7] with eight axiom schemes and modus ponens) and to enrich it with the prelinearity scheme $(A \rightarrow B) \vee(B \rightarrow A)$. Let us denote the resulting calculus again by the letter $G$. The fact that the calculus $G$ is complete w.r.t. the standard G-algebra was proved by M. Dummett in [4]; the logic G is sometimes also called Dummett logic.

A semantics for intuitionistic logic can be defined using Kripke models. A Kripke frame for intuitionistic logic is a structure $\langle W, R\rangle$ where $R$ is an ordering on a (nonempty) set $W$. The relation $R$ is called an accessibility relation of the frame $\langle W, R\rangle$. A Kripke model for intuitionistic logic is a triple $\langle W, R, \|-\rangle$ such that $\langle W, R\rangle$ is a Kripke frame and $\|-$ (a forcing relation) is a relation between elements of $W$ and propositional formulas satisfying the following conditions for all $x, y \in W$, all propositional formulas $A$ and $B$ and all propositional atoms $p$ :

- if $x R y$ and $x \|-p$ then $y \|-p$

- $x \|-A \& B$ iff $x \|-A$ and $x \|-B$

- $x \|-A \vee B$ iff $x \|-A$ or $x \|-B$

- $x \|-\neg A$ iff $y \|+A$ for all $y$ such that $x R y$

- $x \|-A \rightarrow B$ iff $y \|+A$ or $y \|-B$ for all $y$ such that $x R y$.

The first condition is called a persistency condition. This condition is easily shown to be true for all formulas, not just atoms. A Kripke model $\langle W, R, \|-\rangle$ is a counter-model for a formula $A$ if $x \|+A$ for some $x \in W$. A formula $A$ is an intuitionistic tautology if it has no counter-model. For more about Kripke semantics for intuitionistic logic see e.g. [3].

The following definition and lemma show that Kripke models can be useful also in connection with Gödel fuzzy logic. A Kripke frame $\langle W, R\rangle$ is connected if $x R y$ or $y R x$ whenever there is a $z$ such that $z R x$ and $z R y$. Otherwise speaking, $\langle W, R\rangle$ is connected if the set of all elements of $W$ accessible from any $z$ is linearly ordered.

Lemma 1 The Gödel fuzzy logic $\mathrm{G}$ is sound w.r.t. the class of all Kripke models $\langle W, R, \|-\rangle$ whose frame $\langle W, R\rangle$ is connected.

Proof If $x \| f(A \rightarrow B) \vee(B \rightarrow A)$ then, by the definition of Kripke model, there exist $y_{1}$ and $y_{2}$ such that $x R y_{1}, x R y_{2}, y_{1}\left\|-A, y_{1}\right\|+B, y_{2} \|-B$, 

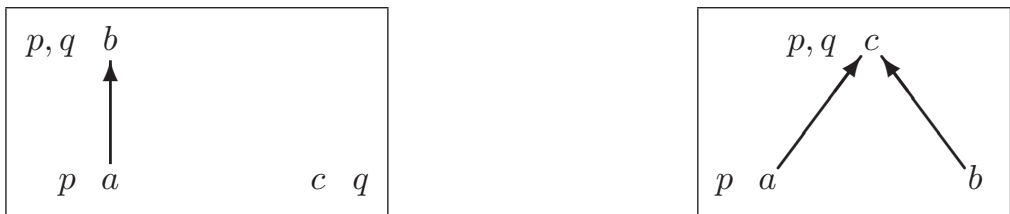

Figure 1: Kripke models ...

$y_{2} \|+A$. By the persistency condition, $y_{1}$ and $y_{2}$ must be incomparable. Thus the prelinearity scheme can be violated only in a model the frame of which is not connected. The rest is soundness of intuitionistic logic with respect to Kripke models (of any kind). QED

Let $A$ be a formula unprovable in $\mathrm{G}$. By completeness of $\mathrm{G}$ w.r.t. the standard G-algebra $[0,1]_{\mathrm{G}}$ (i.e. by the Dummett's result mentioned above) there exists a truth evaluation $e$ (i.e. a function from propositional atoms to $[0,1]$ ) such that $e(A)<1$. Let $V$ be the set of all truth values of atoms occuring in $A$ (i.e. $V$ is the range of the restriction of $e$ to atoms in $A$ ), put $W=V \cup\{0,1\}$. For $x, y \in W$, say that $x R y$ if $y \leq x$ in the sense of the ordering of $[0,1]$. Put $x \|-p$ if $x \leq e(p)$. A straightforward induction on complexity of $B$ shows $x \|-B \Leftrightarrow x \leq v(B)$ for any $x \in W$ and any formula $B$ not containing other propositional atoms than those occuring in the original formula $A$. Since $e(A)<1$, we have $1 \|+A$. Thus $\langle W, R, \|-\rangle$ is a Kripke counter-model for $A$.

The above argument shows that the logic $\mathrm{G}$ is complete w.r.t. the class of all (finite) linearly ordered Kripke models. For our purposes, however, this argument is somewhat misleading because it may suggest that (connected) Kripke models which are not linearly ordered are useless when thinking about the logic G. Indeed they are useless for the purpose of showing that a given formula is not a G-tautology. But our point is to show that they may be useful for other purposes.

Let $\mathbf{K}=\langle W, R, \|-\rangle$ be a Kripke model. Let $\mathrm{D}_{\mathbf{K}}(A)=\{x \in W ; x \|-A\}$. We say that $A$ defines the set $\mathrm{D}_{\mathbf{K}}(A)$. A set $X \subseteq W$ is definable if $X=\mathrm{D}_{\mathbf{K}}(A)$ for some formula $A$. Sometimes we shall write only $\mathrm{D}(A)$ instead of $\mathrm{D}_{\mathbf{K}}(A)$. Note that any definable set $X$ is upwards closed, i.e. $y \in X$ whenever $x \in X$ and $x \leq y{ }^{1}$

Lemma 2 Let $\mathbf{K}=\langle W, R, \|-\rangle$ be any Kripke model. If $\mathrm{G} \vdash A \rightarrow B$ then $\mathrm{D}_{\mathbf{K}}(A) \subseteq \mathrm{D}_{\mathbf{K}}(B)$. If $A$ and $B$ are $\mathrm{G}$-equivalent then $\mathrm{D}_{\mathbf{K}}(A)=\mathrm{D}_{\mathbf{K}}(B)$.

Proof Obvious.

\footnotetext{
${ }^{1}$ The fact that we use the word "definable" as a technical term is the reason why we have decided to speak about "inter-expressibility", rather than "inter-definability", of logical connectives.
} 
Theorem 1 The formula $p \& q$ is not G-equivalent to any formula built up from $p$ and $q$ using only $\rightarrow, \vee$ and $\neg$. The formula $p \rightarrow q$ is not $\mathrm{G}$-equivalent to any formula built up from $p$ and $q$ using only $\&, \vee$ and $\neg$. Thus, in the logic $G$, neither conjunction nor implication is expressible in terms of the remaining three logical connectives.

Proof Consider the Kripke model in the left of Fig. 1. Its domain is $\{a, b, c\}$. Besides the pair $[a, b]$ indicated by an arrow, its accessibility relation contains also three non-indicated elements $[a, a],[b, b]$, and $[c, c]$. The model is connected. The atom $p$ defines the set $\{a, b\}$ and the atom $q$ defines the set $\{b, c\}$. The formula $p \& q$ defines the set $\{b\}$. We claim and prove by induction the following: if $A$ contains no conjunction and $b \in \mathrm{D}(A)$ then $a \in \mathrm{D}(A)$ or $c \in \mathrm{D}(A)$. Thus no $A$ not containing \& can define the set $\{b\}$ and hence no such formula is equivalent to $p \& q$.

Let us show the induction step for implication, the remaining cases are similar or simpler. Let $A$ be $B \rightarrow C$ and let $b \in \mathrm{D}(A)$. So $b \notin \mathrm{D}(B)$ or $b \in \mathrm{D}(C)$. If $b \notin \mathrm{D}(B)$ then, by the definition of Kripke model, we have $a \notin \mathrm{D}(B)$ and $\{a, b\} \subseteq \mathrm{D}(B \rightarrow C)$. If $b \in \mathrm{D}(C)$ then, by the induction hypothesis for $C$, $a \in \mathrm{D}(C)$ or $c \in \mathrm{D}(C)$. This is sufficient since $\mathrm{D}(C) \subseteq \mathrm{D}(B \rightarrow C)$.

Similar argument shows that, in the model on the right hand side of Fig. 1, the formula $p \rightarrow q$ defines the set $\{b, c\}$ while no formula not containing implication can define the same set. QED

We close the paper with some remarks. First, the fact that in intuitionistic logic conjunction and implication is not expressible in terms of the remaining connectives is known for a long time and is thoroughly elaborated e.g. in [2]. The models in Fig. 1 are, with some modifications, taken from [2]. These models work also for intuitionistic logic.

Second, the question whether conjunction or implication is G-expressible in terms of the remaining connectives was raised in [1], where a partial result was proved: in G, conjunction is not expressible using only implication and negation.

Third, using the method of definable sets in Kripke models one can show that in intuitionistic logic disjunction in not expressible in terms of the remaining connectives. On the other hand, in Gödel fuzzy logic disjunction is expressible in terms of conjunction and implication, see [1].

Our fourth and final remark is for readers who prefer thinking about truth values to thinking about Kripke models. With any Kripke model $\langle W, R, \|-\rangle$, a Heyting algebra $\mathbf{H}$ is naturally associated: the domain $H$ of $\mathbf{H}$ are the definable subsets of $W$, the extremal truth values $1_{\mathbf{H}}$ and $0_{\mathbf{H}}$ are $W$ and $\emptyset$ respectively, and the operations of $\mathbf{H}$ are $\cup, \cap$ and the residuum function $\Rightarrow$ defined by $X \Rightarrow Y=\bigcup\{Z \in H ; Z \cap X \subseteq Y\}$. For example, the left model in Fig. 1 has six definable sets, namely $\{a, b, c\},\{a, b\},\{b, c\},\{b\},\{c\}$ and $\emptyset$ (exercise: which formula defines the set $\{c\}$ ?). A Heyting algebra $\mathbf{H}=\langle H, \wedge, \vee, \Rightarrow, 0,1\rangle$ is said 

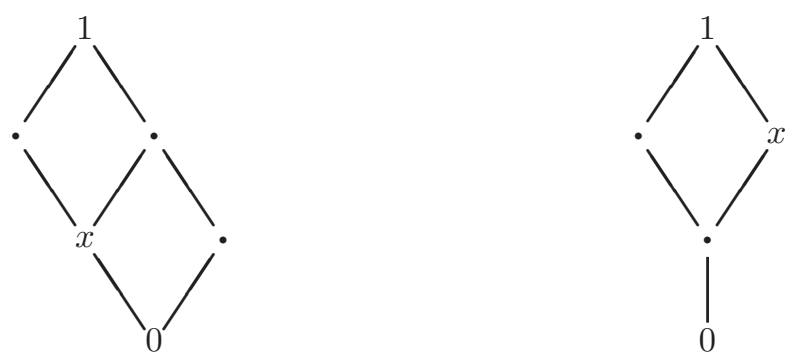

Figure 2: $\ldots$ and corresponding Heyting algebras

to be a G-algebra if it satisfies the equality $(x \Rightarrow y) \vee(y \Rightarrow x)=1$ for all $x$ and $y$. The Heyting algebras corresponding to the models from Fig. 1 are on Fig. 2. Both are G-algebras. In the left algebra the element $x$ can be obtained from some remaining elements by $\wedge$ but cannot be obtained from remaining elements (and 0 and 1 ) using $\vee$ and $\Rightarrow$. In the right algebra the element $x$ can be obtained by $\Rightarrow$ but cannot be obtained from the remaining elements using $\wedge, \vee$ and the negation function $x \mapsto(x \Rightarrow 0)$.

\section{References}

[1] K. Bendová. A note on Gödel fuzzy logic. Soft Computing, 2(4):167-167, 1999.

[2] P. Burdová. Některé sémantické metody v intuicionistické logice (Some Semantical Methods in Intuitionistic Logic). Master's thesis, Philosophical Faculty of Charles University, Department of Logic, 1998.

[3] D. van Dalen. Intuitionistic logic. In D. M. Gabbay and F. Guenthner, editors, Handbook of Philosophical Logic, number 164-167 in Synthese Library, chapter III.4, pages 225-340. Kluwer, Dordrecht, 1986.

[4] M. Dummett. A propositional calculus with denumerable matrix. J. Symb. Logic, 25:97-106, 1959.

[5] K. Gödel. Zum intuitionistischen Aussagenkalkül. Anzeiger Akademie der Wissenschaften Wien, Math.-naturwissensch. Klasse, 69:65-66, 1932. Also in Ergebnisse eines matematischen Kolloquiums 4 (1933), 40.

[6] P. Hájek. Metamathematics of Fuzzy Logic. Kluwer, 1998.

[7] S. C. Kleene. Introduction to Metamathematics. D. van Nostrand, 1952. 